\title{
DNAJs: more than substrate delivery to HSPA
}

\author{
Suzanne L. Dekker, Harm H. Kampinga and Steven Bergink* \\ Department of Cell Biology, University Medical Center Groningen, University of Groningen, Groningen, Netherlands
}

\section{OPEN ACCESS}

Edited by:

Pierre Goloubinoff

University of Lausanne, Switzerland

Reviewed by:

Nico P. Dantuma,

Karolinska Institutet, Sweden

Krzysztof Liberek,

University of Gdansk, Poland

Pierre Genevaux,

Centre National de la Recherche

Scientifique, France

*Correspondence:

Steven Bergink

Department of Cell Biology, FB30, University Medical Center Groningen,

University of Groningen, Antonius

Deusinglaan 1, Groningen 9700AD,

Netherlands

s.bergink@umcg.nl

Specialty section:

This article was submitted to

Protein Folding, Misfolding and

Degradation,

a section of the journal

Frontiers in Molecular Biosciences

Received: 30 April 2015

Accepted: 12 June 2015

Published: 30 June 2015

Citation:

Dekker SL, Kampinga HH and Bergink $S$ (2015) DNAJs: more than substrate delivery to HSPA.

Front. Mol. Biosci. 2:35.

doi: 10.3389/fmolb.2015.00035
Proteins are essential components of cellular life, as building blocks, but also to guide and execute all cellular processes. Proteins require a three-dimensional folding, which is constantly being challenged by their environment. Challenges including elevated temperatures or redox changes can alter this fold and result in misfolding of proteins or even aggregation. Cells are equipped with several pathways that can deal with protein stress. Together, these pathways are referred to as the protein quality control network. The network comprises degradation and (re)folding pathways that are intertwined due to the sharing of components and by the overlap in affinity for substrates. Here, we will give examples of this sharing and intertwinement of protein degradation and protein folding and discuss how the fate of a substrate is determined. We will focus on the ubiquitylation of substrates and the role of Hsp70 co-chaperones of the DNAJ class in this process.

Keywords: DNAJ, ubiquitin E3 ligases, HSP70 heat-shock proteins, degradation, protein folding

\section{Protein Stress}

The capacity of the protein quality control network is limited and collapse occurs when the system is overwhelmed. This can occur after sudden massive stress including temperature shifts, nutrient deprivation or pathogenic infection (Lee, 1992; Scheuner et al., 2001; Kaufman et al., 2002; Schelhaas et al., 2007; Morimoto, 2008). These types of stresses are usually sensed and the organism responds with the activation of stress pathways, such as the heat shock response after temperature elevation (Morimoto, 2008).

Also "normal" conditions in the cell, for example the increased synthesis of proteins during cell cycle progression (Morimoto, 2008) can be considered as physiological protein stress requiring increased folding capacity and adaptations in the quality control network. The difference between these two types of protein stress is that the latter is enlisted and that substrate recognition and fate are pre-determined, for example cyclins are degraded upon orchestrated phosphorylation events (Murray, 2004). In contrast, sudden or accidental protein stress is unpredictable and requires a system that can recognize the client(s), which lack(s) a common motif that is usually present in the regular enlisted clients. Typically, these accidental clients become unfolded after stress or are intrinsically misfolded (i.e., genetically encoded).

\section{Protein Stress and Its Impact on Human Pathology}

Protein stress can accumulate over time as well, as is the case in many diseases that are associated with an accumulation of misfolded proteins. Often this type of slowly accumulating protein damage is-initially - not triggering a strong stress signaling, but remains undetected. Yet, this type of stress may also overwhelm the protein quality control network and cause a similar collapse, ultimately 
resulting in the associated pathology. Diseases driven by protein stress range from cataract, type II diabetes, atrial amyloidosis to neurodegenerative diseases (Chiti and Dobson, 2006).

Moreover, it is known that hypomorphic mutations, a partial loss of gene function, can lead to an accelerated phenotype in the background of a compromised protein quality control network (Ben-Zvi et al., 2009). Suggesting that genome alterations can lead to metastable or aberrant polypeptides that stochastically accumulate in time. If true, the source of protein stress would expand to single nucleotide polymorphisms (SNPs) and somatic mutations that slowly accumulate as we age.

\section{Molecular Chaperones}

Heat Shock Proteins (HSPs) play a central role in protein homeostasis and are upregulated by the diverse protein stress signaling pathways in cells under many conditions, most prominently acute stress, that challenge protein homeostasis (Morimoto, 2008). For various HSPs, it has been established that they have so-called molecular chaperone activity. Usually, this is associated with folding nascent chains into their native state or refolding of stress-unfolded proteins. However, their activities are also needed for degradation of misfolded or mutant proteins and even for remodeling of active protein complexes. As a matter of fact, the most ancient members of the molecular chaperones, DnaJ, and DnaK (Hsp70/HSPA), were originally identified as essential components controlling (dis)assembly of phage lambda replication complexes (Konieczny and Zylicz, 1999).

A wide network of molecular chaperones exists that encompasses the chaperonins, the HSPAs (Hsp70s and Hsc70s, eleven in humans) with its co-chaperones: the DNAJs (50 members in humans) and the Nucleotide Exchange factors (NEF, 13 members in humans), the HSPBs (small HSPs, 10 members in humans) and the HSPCs (Hsp90s, five members in humans) (Kampinga and Craig, 2010). Here we will focus on the DNAJs in the HSPA system (Figure 1A), as these are involved in substrate recognition and seem to function at the crossroad of folding and degradation.
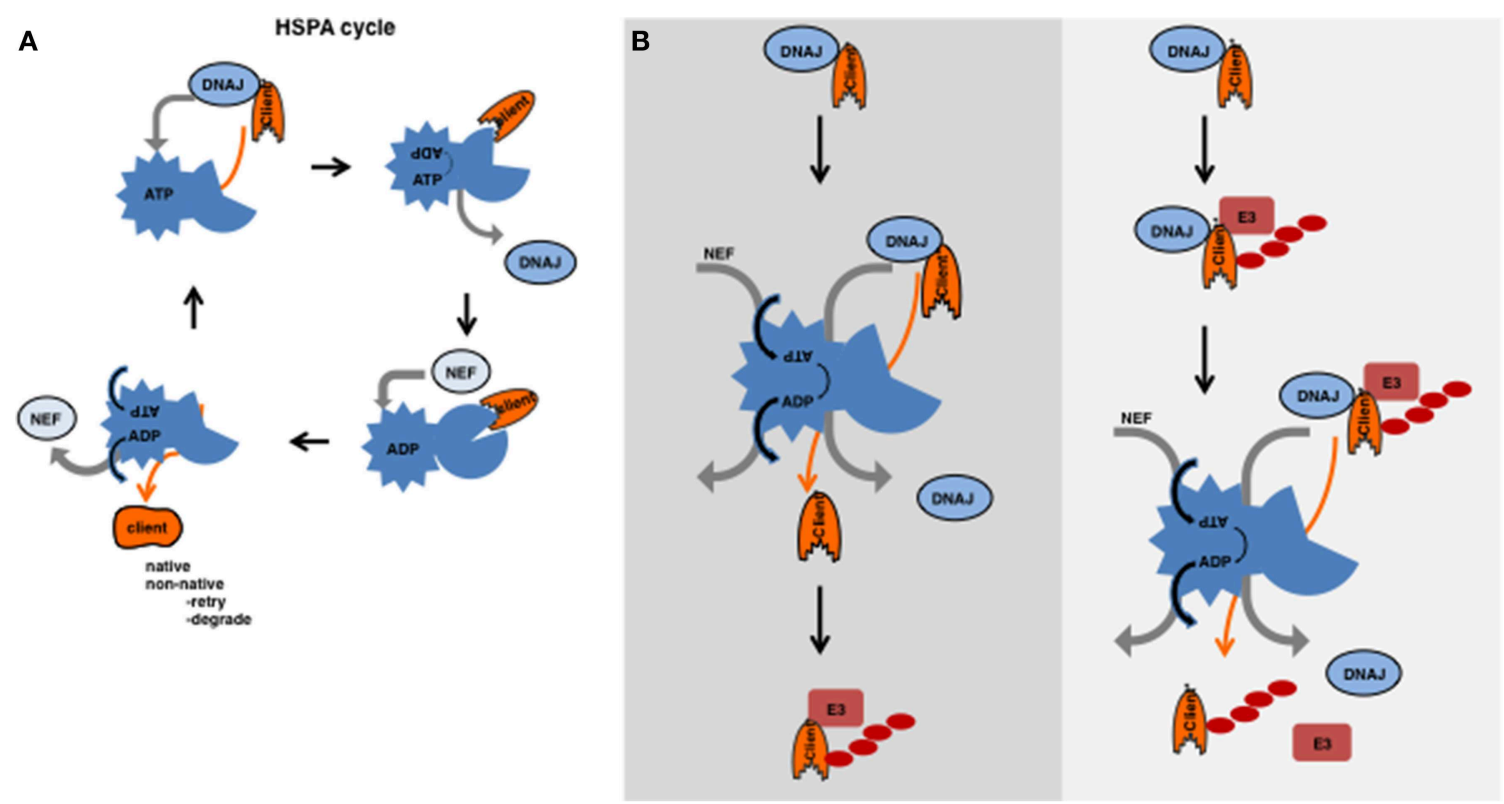

FIGURE 1 | (A) HSPA cycle. HSPAs (HSP70s and HSC70s) contain an $\mathrm{N}$-terminal ATPase domain connected by a hydrophobic linker to a variable C-terminal peptide-binding domain (Hartl et al., 2011). This peptide-binding domain binds to a stretch of hydrophobic residues flanked by positive residues, which is predicted to occur every 40 amino acids (Frydman et al., 1999). In the folded state, these hydrophobic residues are buried inside, but are exposed in the unfolded or misfolded state. The energy obtained from the hydrolysis of ATP is required for assisted folding. However, the exact mechanism how an HSPA supports folding is still not yet completely understood. HSPA functions with the help of co-chaperones that orchestrate the cycle of ATP hydrolysis and substrate/client binding and release. DNAJs recognize clients and subsequently bind to the ATP-bound form of HSPA; upon binding of the DNAJ-client complex, ATP is hydrolysed by the HSPA and the DNAJ is released. Upon hydrolysis and DNAJ release, the peptide-binding-domain of HSPA undergoes a conformational change and clams around the polypeptide (the substrate) (Jiang et al., 2007; Swain et al., 2007; Bertelsen et al., 2009). The NEF has affinity to the ADP-bound form and mediates the exchange of ADP for ATP. The client has less affinity for the ATP-bound form of HSPA and releases together with the NEF. As a result, the client can fold or will re-enter the cycle if not completely folded, or somehow can be transferred to degradation machineries. (B) Substrates ubiquitylated before or after the action of HSPA. On the left, the canonical model is depicted in which an intrinsically unstable substrate is first recognized by a DNAJ then transferred to the HSPA cycle and after a futile folding event ubiquitylated and subsequently degraded. On the right, a model is presented in which an unfolded substrate is recognized by a DNAJ after which an E3 ligase can ubiquitylate it and the HSPA cycle acts on the ubiquitylated substrate. After the action of HSPA, the substrate is liberated from both the DNAJ and the E3 ligase (complex) and targeted for degradation. 


\section{The DNAJs: A Crucial Role in Protein Quality Control}

The HSPAs function with the help of several co-chaperones and collaborate with the other chaperones. The most important co-chaperones are the DNAJs and the NEFs. DNAJs recognize substrates/clients and subsequently bind to the ATP-bound form of HSPA (for the HSPA cycle see Figure 1A).

DNAJs are defined by the presence of a J-domain, an approximately 70 amino acid domain that consist of four alpha helices and an accessible loop to which the (ATP-bound) ATPase domain of HSPA binds (Jiang et al., 2007). In this loop lies a Histidine Proline Aspartate (HPD) motif, which is crucial for binding to HSPAs. DNAJs are divided based on their domain structure into three classes, DNAJAs, DNAJBs and DNAJCs (Kampinga and Craig, 2010). Briefly, in both DNAJAs and DNAJBs, the N-terminal J domain is followed by a Glycine/Phenylalanine-rich stretch. In the DNAJA class proteins, two domains (CTDI and CTDII) are located at the Cterminus that are involved in substrate recognition by forming a hydrophobic pocket that binds to the substrate (Li et al., 2003). The first of these CTDs contains a zinc finger. The $\mathrm{C}$ terminus in the DNAJB lacks the organization into two CTDs or and more specifically contains no zinc finger and specific information of substrate binding regions is still lacking. The DNAJC class comprises any J-domain containing protein that does not fit in the A or B class.

A wide variety of domains can be found in the different $J$ proteins. Many of these are known or thought to be involved in substrate interactions, thus a division based on substrate recognition has been proposed as well (Kampinga and Craig, 2010). Many J-proteins are believed to have a promiscuous client binding (mostly from class A and B and a few from class C) while others seem to display high substrate specificity (mostly class C).

\section{Interplay between Degradation and Folding}

The notion that there are around 50 DNAJs, 11 HSPAs, and $13 \mathrm{NEF}$ at least suggest a high potential of many possible combinations, theoretically resulting in flexibility and a broad substrate range. Some DNAJs and HSPAs are localized to specific organelles and seem to form combinations with the compartment specific Hsp70 (HSPA) members, but for other (cytosolic) HSPAs it has been shown that they can indeed operate with different DNAJs (Kampinga and Craig, 2010). The interplay between the HSPA cycle and the degradation machineries makes matters even more complex. The idea that the fate of a protein is determined by this interplay of the chaperone systems and the degradation systems is often referred to as the triage decision (Connell et al., 2001; Houck et al., 2012). The classical view is that the cell first attempts to (re)fold the damaged or metastable protein. If folding fails, the cell will then attempt to degrade it (Figure 1B). Many proteins that are part of the folding machinery interact with the cellular degradation machinery to make this transition smoothly. For example, DNAJB2 interacts with ubiquitylated proteins via its ubiquitin interacting motifs (UIM) (Westhoff et al., 2005) and
Bag1 (a NEF) contains a ubiquitin-like (UBL) domain which are known to interact with subunits of the proteasome (Lüders et al., 2000). An alternative view would be that degradation and (re)folding act in parallel and that chaperones are an integral part of the degradation machinery and have a different function besides folding. These scenarios are not mutually exclusive and, depending on the substrate, both might happen.

How the HSPA machinery selects and recognizes the different types of substrates and which parameters determine the fate of the clients (folding, degradation or aggregation) are still open questions. Here we will give examples of clients that are dealt with by different combinations of DNAJs and HSPAs. Moreover, we will discuss the recent insights in the interplay between the HSPA cycle and the ubiquitin proteasome system (UPS) (Box 1).

\section{De Novo Protein Folding}

The folding of newly synthesized proteins is different from refolding reactions, partly due to the vectorial nature of translation. Indeed, early studies clearly demonstrated that nascent chain folding and refolding require different sets of chaperones (Frydman et al., 1994, 1999). Folding of newly synthesized proteins can occur co-translationally and sometimes also post-translationally. In prokaryotes, folding involves many different partners including trigger factor which prevents misfolding of nascent chains by delaying protein folding (Agashe et al., 2004). Although far from completely resolved, in cotranslational folding, the different domains fold separately. As often, the fundamental studies in yeast have shed light on this process (Albanèse et al., 2006). Two complexes are associated with the ribosome and the emerging polypeptide. First, the nascent polypeptide associated complex (NAC), a heterodimer that consists of an alpha and a beta subunit, binds to all nascent chains. Second, a set of Hsp70s (Ssb1 and 2) is bound to translating ribosomes, which function together with the DNAJ Zuol and the NEF Sse1 and bind to the polypeptide that extrudes from the ribosomal exit tunnel. Zuol together with Ssz1 (another Hsp70) forms the Ribosome Associated Complex (RAC), and is thought to recruit Ssb1 and 2 to the ribosome. Global identification of Ssb1/2 substrates revealed that especially the more difficult substrates (e.g., longer, several domains, beta sheet enriched, and aggregation prone) are clients (Willmund et al., 2013). Beside Zuo1, two other DNAJ proteins have been identified as being important for nascent chain folding namely Ydj1 and Sis1. These DNAJs function with the "soluble" or cytoplasmic Hsp70s (the Ssa1-4 proteins) and the chaperonin system to guide the final (posttranslational) folding of newly synthesized polypeptides (Kim et al., 2013).

Most of these yeast proteins have mammalian counterparts: Ssz1 is homologous to HSPA14, Zuo1 is homologous to DNAJC2 [also called, Mpp11 (M-phase phosphoprotein 11) (Hundley et al., 2005) or ZRF1 (zuotin related factor 1) (Richly et al., 2010)], Ydj1 is homologous to DNAJA1 and Sis1 is homologous to DNAJB1. HSPA8 is associated with the ribosome and homologous to Ssb2 (Hundley et al., 2005). 


\section{BOX 1 | Protein Degradation.}

The various proteases in the cell are capable of degrading targeted substrates. As a general theme, access to proteases is restricted either due to a high specificity for specific substrates, a common but inducible recognition signal (e.g., the proteasome), or spacious confinement (e.g., the lysosome). The UPS and autophagy are the systems that degrade the bulk of proteins.

UPS

Recognition by the 26S proteasome is done by lysine $(K)$ 48-linked polyubiquitin chains conjugated to substrates that require degradation. Ubiquitin is a polypeptide that is conjugated to target proteins by the subsequent action of three enzymes, an E1 (activating enzyme), an E2 (conjugating enzyme) and an E3 (ligating enzyme). The ligases are responsible for substrate recognition and thus determine the specificity, whereas the E2s usually mediate the conjugation of ubiquitin to the substrate. Occasionally an E4 ligase is required to extend already existing mono- or oligo-ubiquitylation events to polyubiquitin trees. The C-terminal glycine of ubiquitin either forms a peptide bond with the $\mathrm{N}$-terminus of a substrate or an isopeptide bond with the epsilon- $\mathrm{N}$ in internal lysines of the substrate (although conjugation to cysteine and serine have been reported as well). Due to the internal lysines in ubiquitin itself a so-called polyubiquitin tree is build. Polyubiquitylation with a K48 linkage is considered canonical for degradation. Indeed, monoubiquitylation or other types of polyubiquitin trees with different linkage are not necessarily recognized as targeted for destruction. Receptors at the 26S proteasome recognize the polyubiquitin trees, which are cleaved off and recycled. The $26 \mathrm{~S}$ proteasome is a multimeric complex that consists of a $20 \mathrm{~S}$ and $19 \mathrm{~S}$ component. After deubiquitylation, the substrate is unfolded by ATPases associated with various cellular activities (AAA-ATPases) of the 19S, and funneled into the proteolytic chamber (20S). Only when a protein resides in this chamber it is cleaved into peptides.

\section{Autophagy}

Autophagy is a process in which a double membrane-vesicle (the autophagosome) containing the cargo is targeted to, and fused with the lysosome in which the degradation (hydrolysis) takes place. The cargo consists of proteins, protein-complexes, aggregated proteins and even entire organelles. Autophagy is mediated by a set of proteins and includes two types of ubiquitin-like conjugation reactions; the most crucial one is the conjugation of LC3 or ATG8 (in yeast) to phosphoatidylethanolamine at the expanding autophagic membrane. Originally it was thought that autophagy is mostly nonspecific and just randomly engulfs cytoplasm. However, the current dogma is that most autophagy is in fact specific. Again, ubiquitylation can form the signal that is recognized by specific receptors that both bind polyubiquitylated substrates and LC3, the double membrane then encloses the cargo and translocates to the lysosome.

\section{Quality Control at the Ribosome}

Translational errors may occur due to aberrant mRNAs, misfolding due to intrinsic properties of the nascent chain itself or to other events that lead to stalling of the translational machinery. As a general theme, these errors are sensed during translation and the emerging polypeptide is co-translationally ubiquitylated, released and degraded. Depending on the type of translational problem and substrate, different chaperones and components of the UPS are involved. For example, long stretches of basic amino acids are recognized by the NAC complex and ubiquitylated by the E3 ligase Not4 (Dimitrova et al., 2009). Other types of translational difficulties, such as the translation of the polyA sequence that leads to pausing, require another E3 ligase, namely Ltn1. The ubiquitylated polypeptide requires $\mathrm{Cdc} 48^{\mathrm{Ufd} 1 / \mathrm{Npl} 4}$ (p97/VCP in mammalians), a ubiquitin/ubiquitin-like-modifier-specific segregase (Jentsch and Rumpf, 2007), to remove it from the ribosome (Brandman et al., 2012; Defenouillère et al., 2013; Verma et al., 2013). Thus, translational errors usually result in immediate, cotranslational, degradation (Figure 2A) in which chaperones act before ubiquitylation.

Degradation of the emerging polypeptide can in principle always occur at the ribosome, even in the absence of translational errors, as shown by elegant experiments using tandem expressed degradation-reporters (Turner and Varshavsky, 2000). This implies that folding or degradation is an intrinsic fate of the client rather than it is driven by different sets of chaperone machines. In other words, the different ribosome-associated chaperone complexes (in yeast Ssb1/2, RAC, and NAC) prevent aggregation during translation which -depending on the substrate- can result in folding or maintain the substrates/clients accessible to the various E3 ligases that are either present at, or have access to the ribosomes (examples in yeast: Ltn1, Not4, Ubr1).

\section{Chaperones: the Prelude for Ubiquitylation of Proteasomal Clients?}

The idea that unfolded or misfolded clients, similar to nascent chains, need to be kept accessible/soluble in order for a protein quality control E3 ligase to work seems appealing. The idea would be that a chaperone binds first to a potential substrate and keeps this substrate soluble or accessible for the E3 ligase to ubiquitylate this target. The requirement for a chaperone as a recognition or accessibility factor for ubiquitin E3 ligases would not be necessary for regular or enlisted clients (e.g., the cyclins), but only for those that become (partly) unfolded by a random stress event or for those clients that are intrinsically (genetically encoded) misfolded. Chaperones of the HSPB and DNAJ class are well known to be able to recognize these clients and thereby ensure their solubility. In the canonical view, these clients are next transferred to the HSPAs (Hsp70s). Release from the HSPAs occurs via NEFs and interactions with E3 ligases at which level client-fate is determined. However, as we will explain below, evidence is accumulating that other scenarios may also be operational (Figures 1B, 2).

\section{Ubiquitylation and DNAJs in ERAD}

Clear examples where a DNAJ precedes the action of an E3 can be found within ER associated degradation (ERAD). Proteins that are targeted to the ER, but are unwanted because they are intrinsically unstable, aggregation prone or unfolded, are cleared via the ERAD pathway. For ER luminal and transmembrane clients, DNAJs (DNAJB11, DNAJB9, and DNAJC10 also known as Erdj3, -4, and -5), recognize misfolded clients that subsequently are ubiquitylated by the membrane embedded E3 ligases Hrd1 or RMA1 and the E4 ligase gp78 (Buchberger et al., 2010). For those clients that expose their unstable region to the cytosol, soluble-DNAJs play a role in 


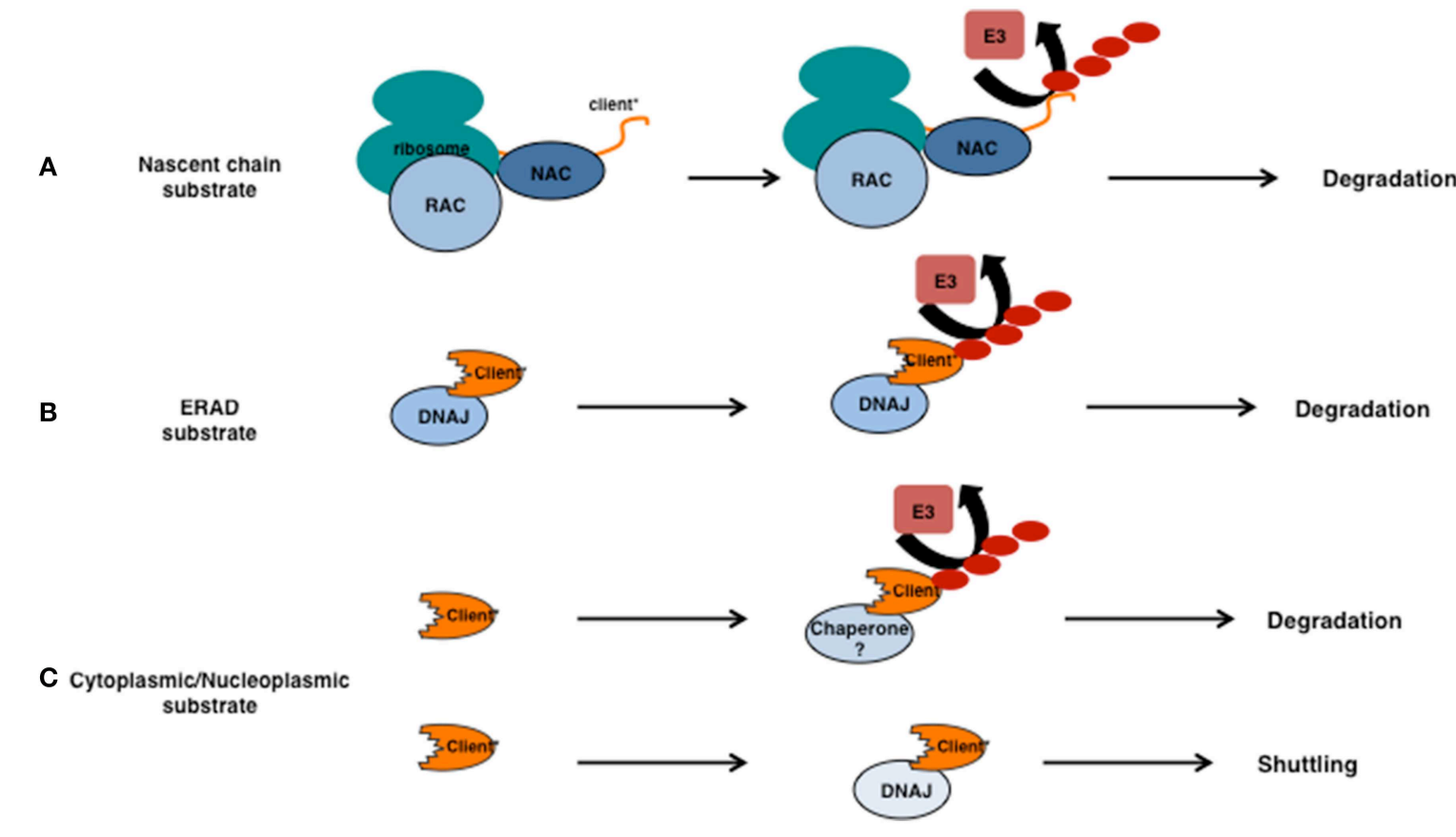

FIGURE 2 | Involvement for chaperones in substrate ubiquitylation. (A) The relationship between chaperones and ubiquitin E3 ligases for unstable nascent chains. Unstable nascent chains are bound by the chaperone systems present at the ribosome (here the NAC and RAC) and ubiquitylation of the emerging polypeptide happens co-translationally. (B) ERAD substrates that are intrinsically unstable (indicated by the *) are recognized by DNAJs prior their ubiquitylation and retrotranslocation. (C) If cytoplasmic or nucleoplasmic substrates require chaperones prior their ubiquitylation is currently unclear. Sis1/DNAJB1 is required for the nuclear relocalization of certain unstable substrates in an ubiquitin-independent manner. conjunction with the E3 ligase Doa10 (Ravid et al., 2006). After or during ubiquitylation, all ERAD clients require retrotranslocation over, or dislocation from the lipid bilayer, a process that is mediated by the $\mathrm{p} 97 / \mathrm{Cdc} 48^{\mathrm{Ufd} 1 / \mathrm{Np} 14}$ complex (Tsai et al., 2002).

Also for integrated membrane proteins, DNAJs may act prior to E3 ligases in degradation. Several model substrates exist exposing their unstable region to the cytosol and for some of these the relationship between chaperone function and ubiquitylation status has been determined. For example degradation and ubiquitylation of the Doa10 substrates Ste6* and PMA1* depends on the cytosolic Hsp70s (Ssa1-4) and the (cytosolic) DNAJs Ydj1 and Hlj1 (Han et al., 2007; Nakatsukasa et al., 2008) (Figure 2B). Other DNAJs are also involved in Doa10-mediated substrate ubiquitylation as Sis1, but not the cytosolic Hsp70s (Ssa1-4), is required to ubiquitylate the degradation prone part from the kinetochore protein $\mathrm{Ndc10}$. Instead, Hsp70s are required to prevent aggregation of the ubiquitylated client (Shiber et al., 2013) and clearly function after ubiquitylation. These results are in line with the idea that E3 ligases involved in clearing misfolded substrates require a molecular chaperone for their functioning (Figure 2).

However, for other substrates (e.g., soluble targets of the protein quality control E3 ligases Ubr1 and San1) Sis1 (or Ydj1) is not a prerequisite for ubiquitylation but is essential for efficient client degradation (Park et al., 2013; Mehnert et al., 2015; Miller et al., 2015) and thus seem to play a role downstream of ubiquitylation.

\section{Ubiquitylation and DNAJs of Cytoplasmic and Nucleoplasmic Clients}

Whereas, translation quality control and ERAD are vectorial by nature, the organization of quality control over soluble proteins is not. If the soluble (stress induced or accidentally misfolded) clients in the cytosol and nucleoplasm also require a chaperone prior ubiquitylation is currently unclear. The notion that overexpression of certain DNAJs accelerates degradation of substrates is suggestive of a direct impact of DNAJs on degradation/ubiquitylation (Westhoff et al., 2005). However, clear evidence that DNAJs or HSPBs are required for ubiquitylation of these accidental clients is currently lacking (Figure 2C). Deletion of individual DNAJs or other molecular chaperones typically does not result in accumulation of their unmodified clients. It is not unlikely that there is functional redundancy between the different and numerous chaperones (e.g., DNAJs) in these compartments making the fate of substrates/clients harder to study. Moreover, to distinguish this process from translational protein quality control (Wang et al., 2013), a substrate that becomes unstable after translation vs. a constitutively unstable substrate, is required. For example a substrate that after the addition of a specific small molecule triggers unfolding of that substrate would be a great asset. This type of substrate would enable the study of posttranslational protein quality control under normal, non-stressed, conditions. This type of research is especially important as, 
in many of the aforementioned diseases, protein stress occurs post-translationally.

\section{Nuclear Transport of Cytosolic Substrates}

As mentioned above, ERAD substrates are degraded in the cytoplasm and retro-translocation is necessary as the proteasome is not present in the ER. One might suspect that degradation of cytoplasmic clients is more straightforward as the proteasome is present in the same compartment. However, recent evidence suggests that, at least for some substrates, this is not the case. Instead, cytosolic model substrates were found to shuttle to the nucleus for degradation (Figure 2C) (Heck et al., 2010; Prasad et al., 2010; Park et al., 2013). This transfer across the nuclear envelope depends on the DNAJ Sis1 (DNAJB1 in humans) (Park et al., 2013). Increasing the proteotoxic stress hampers this process, probably due to a sequestration of Sis1 to other clients, and results in a reduction in degradation of these substrates (Park et al., 2013). Transport of un- or misfolded substrates to a (juxta)nucleolar localization seems to be a more general feature for both nuclear (Nollen et al., 2001) and cytosolic proteins (Miller et al., 2015). Targeting of substrates to these structures depends on, again, Sis1 and the v-snare protein Btn2 (Malinovska et al., 2012; Miller et al., 2015). Whether the nucleolar accumulation per se determines the fate of the client is, however, unlikely as the accumulation in the nucleolus also occurs to nuclear clients that require refolding after heat stress (Nollen et al., 2001). Here, the accumulation of substrates in the nucleolus is reversible and accompanied by a strong sequestering of, normally cytosolic, HSPA1A (Hsp70) to the nucleolus. In line, ubiquitylation does not seem to play a role in the transfer across the nuclear envelope (Park et al., 2013; Miller et al., 2015).

What remains a question is how cytoplasmic proteins cross the nuclear barrier and whether or not posttranslational modifications play a role in this cytoplasmic-nuclear shuttling. Post-translational modifications as SUMOylation (Small Ubiquitin-like Modifier) and poly(ADP-ribosyl)ation have been implemented in protein stress responses (Haince et al., 2006; Golebiowski et al., 2009), the formation of stress bodies (Nagai et al., 2011) and also in nucleolar integrity (Finkbeiner et al., 2011; Boamah et al., 2012).

Why the nucleolus would serve as storage for proteins before folding or degradation has still remained enigmatic. Even more so, one may wonder why cytosolic substrates are translocated to the nucleus/nucleoli for degradation, given that in the cytosol proteasomes are fully active and autophagy is present. Notably, in all of these experiments the substrates used are engineered proteasomal clients. The transfer of these clients across the nuclear pore could therefore be an extension of the aforementioned ribosomal quality control mechanism, which indeed involves Sis1, and that shuttling for these substrates may occur co-translationally. However, adding an nuclear localization signal increases the degradation speed significantly (whereas adding a NES inhibits it) (Park et al., 2013), suggesting that the nuclear shuttling of cytoplasmic substrates is not that efficient and probably not coupled to translation.
One could hypothesize that substrates are normally already shuttling between the cytosol and nucleus and that degradation is simply faster in the nucleus in which the proteasomal concentration is higher (Russell et al., 1999). However, this would suggest that the capacity of the UPS in the cytoplasm is rate limiting, which seems not to be the case (Park et al., 2013). So, shuttling of un- or mis-folded clients to the nuclear/nucleolar compartment indeed seems an active, regulated process. Perhaps the cytosolic compartment, or the organelles herein, are more vulnerable to proteotoxic stress and the re-localization to the nucleus/nucleolus serves to avoid immediate cellular arrest or death. However, given the complex processes that take place in the nucleus, and the presence of the genetically vulnerable rDNA in the nucleolus, this might hamper nuclear processes such as transcription and replication.

\section{CFTR; Escaping Initial Quality Control Is Not Enough}

One substrate that illustrates the complexity of quality control is the disease-associated form of cystic fibrosis transmembrane conductance regulator (CFTR) (Younger et al., 2006; Okiyoneda et al., 2010). For some of the CFTR mutations, in particular the deletion of phenylalanine at position $508\left(\right.$ CFTR $^{\text {delta508}}$ ) (Lukacs and Verkman, 2012), premature degradation is thought to underlay CF-pathology. This deletion destabilizes or kinetically traps CFTR, which targets this protein to the ERAD pathway. Numerous chaperones, including DNAJs and HSPAs, and E3 ligases have been implicated in the clearance of mutant CFTR. CFTR $^{\text {delta508 }}$ is ubiquitylated either by the Hrd1 E3 ligase complex or the Rmal/Derlin1 E3 ligase complex and the GP78 E4 ligase in a process that is enabled by the membrane embedded DNAJB12, after which retro-translocation by $\mathrm{p} 97$ and degradation occurs (Younger et al., 2006; Grove et al., 2011).

Skipping this initial ERAD quality control enables part of the protein to arrive at the plasma membrane to fulfill its function (Kälin et al., 1999). Unfortunately, this rescued mutant protein is still degraded, now by a different set of chaperones and E3 ligases, involving DNAJA1, DNAJB2, HSPA8, HSPA1A, Bag1, and the E3 ligase CHIP (C-terminal Hsc70/HSPA8 interacting protein) (Meacham et al., 2001; Okiyoneda et al., 2010). CHIP has a well-known function in cytoplasmic protein quality control, it binds directly to HSPA and is involved in ubiquitylating HSPA clients that need to be degraded. The work on CFTR ${ }^{\text {delta508 }}$ nicely illustrates that each cellular compartment has a different set of protein quality control components. Moreover, it points out that in the lifetime of a protein it encounters various and different quality checks.

\section{Summary}

The examples of substrates in the ERAD pathway and protein quality control during translation illustrated that for certain substrates a molecular chaperone (a DNAJ) is required for ubiquitylation while this is unclear for others.

The "holdase" function of DNAJs is well suited to assist E3 ligase function for those substrates that are intrinsically 
unstable and thus would misfold, form aggregates or fibrils in the absence of a molecular chaperone. Intriguingly, for some of these substrates HSPAs are necessary for degradation but not for ubiquitylation, indicating that they act after the E3 ligase but before proteolysis. Perhaps to prevent aggregation in the brief moment a substrate is delivered to the proteasome. Instead, the energy provided by the ATP hydroslysis of Hsp70s could also facilitate the release of the DNAJ and the E3 ligase from the ubiquitylated substrate (Figure 1B right panel). This un-entanglement-function of HSPA would not be required for substrates that come from the ER lumen, as both E3 ligase and DNAJ are embedded in the membrane, and retro-translocation is sufficient to clear the substrate from these factors. The view that degradation substrates are immediately marked for destruction without attempts to fold the substrate is not ruling out that futile folding can lead

\section{References}

Agashe, V. R., Guha, S., Chang, H. C., Genevaux, P., Hayer-Hartl, M., Stemp, M., et al. (2004). Function of trigger factor and DnaK in multidomain protein folding: increase in yield at the expense of folding speed. Cell 117, 199-209. doi: 10.1016/S0092-8674(04)00299-5

Albanèse, V., Yam, A. Y. W., Baughman, J., Parnot, C., and Frydman, J. (2006). Systems analyses reveal two chaperone networks with distinct functions in eukaryotic cells. Cell 124, 75-88. doi: 10.1016/j.cell.2005.11.039

Ben-Zvi, A., Miller, E. A., and Morimoto, R. I. (2009). Collapse of proteostasis represents an early molecular event in Caenorhabditis elegans aging. Proc. Natl. Acad. Sci. U.S.A. 106, 14914-14919. doi: 10.1073/pnas.0902882106

Bertelsen, E. B., Chang, L., Gestwicki, J. E., and Zuiderweg, E. R. P. (2009). Solution conformation of wild-type E. coli Hsp70 (DnaK) chaperone complexed with ADP and substrate. Proc. Natl. Acad. Sci. U.S.A. 106, 8471-8476. doi: 10.1073/pnas.0903503106

Boamah, E. K., Kotova, E., Garabedian, M., Jarnik, M., and Tulin, A. V. (2012). Poly(ADP-Ribose) polymerase 1 (PARP-1) regulates ribosomal biogenesis in drosophila nucleoli. PLoS Genet. 8:e1002442. doi: 10.1371/journal.pgen.1002442

Brandman, O., Stewart-Ornstein, J., Wong, D., Larson, A., Williams, C. C., Li, G.-W., et al. (2012). A ribosome-bound quality control complex triggers degradation of nascent peptides and signals translation stress. Cell 151, 1042-1054. doi: 10.1016/j.cell.2012.10.044

Buchberger, A., Bukau, B., and Sommer, T. (2010). Protein quality control in the cytosol and the endoplasmic reticulum: brothers in arms. Mol. Cell 40, 238-252. doi: 10.1016/j.molcel.2010.10.001

Chiti, F., and Dobson, C. M. (2006). Protein misfolding, functional amyloid, and human disease. Annu. Rev. Biochem. 75, 333-366. doi: 10.1146/annurev.biochem.75.101304.123901

Connell, P., Ballinger, C. A., Jiang, J., Wu, Y., Thompson, L. J., Höhfeld, J., et al. (2001). The co-chaperone CHIP regulates protein triage decisions mediated by heat-shock proteins. Nat. Cell Biol. 3, 93-96. doi: 10.1038/35050618

Defenouillère, Q., Yao, Y., Mouaikel, J., Namane, A., Galopier, A., Decourty, L., et al. (2013). Cdc48-associated complex bound to $60 \mathrm{~S}$ particles is required for the clearance of aberrant translation products. Proc. Natl. Acad. Sci. U.S.A. 110, 5046-5051. doi: 10.1073/pnas.1221724110

Dimitrova, L. N., Kuroha, K., Tatematsu, T., and Inada, T. (2009). Nascent peptidedependent translation arrest leads to Not4p-mediated protein degradation by the proteasome. J. Biol. Chem. 284, 10343-10352. doi: 10.1074/jbc.M808840200

Finkbeiner, E., Haindl, M., Raman, N., and Muller, S. (2011). SUMO routes ribosome maturation. Nucleus 2, 527-532. doi: 10.4161/nucl.2.6.17604

Frydman, J., Erdjument-Bromage, H., Tempst, P., and Hartl, F. U. (1999). Cotranslational domain folding as the structural basis for the rapid de novo folding of firefly luciferase. Nat. Struct. Biol. 6, 697-705. doi: 10.1038/10754 to degradation as well (Figure 1A left panel), but indicates that the degradation pathways are even more intertwined with the folding machinery as was previously thought. In that regard, it is not unlikely that the HSPA cycle, besides its canonical role in protein folding, could function to untangle the substrate-E3/E2 ligase complexes for transfer of regular enlisted clients to the proteasome as well (Mehnert et al., 2015).

\section{Acknowledgments}

SD is supported by the Graduate School of Medical Science of the UMCG. HK is supported by a grants from Senter Novem (IOPIGE7004), high Q foundation (project \# 3.1.2), Hersenstichting (project 15F07(2)-58). SB is supported by a Hersenstichting grant (project no. F2013(1)-52).

Frydman, J., Nimmesgern, E., Ohtsuka, K., and Hartl, F. U. (1994). Folding of nascent polypeptide chains in a high molecular mass assembly with molecular chaperones. Nature 370, 111-117. doi: 10.1038/370111a0

Golebiowski, F., Matic, I., Tatham, M. H., Cole, C., Yin, Y., Nakamura, A., et al. (2009). System-wide changes to SUMO modifications in response to heat shock. Sci. Signal. 2, ra24. doi: 10.1126/scisignal.2000282

Grove, D. E., Fan, C.-Y., Ren, H. Y., and Cyr, D. M. (2011). The endoplasmic reticulum-associated Hsp40 DNAJB12 and Hsc70 cooperate to facilitate RMA1 E3-dependent degradation of nascent CFTRDeltaF508. Mol. Biol. Cell 22, 301-314. doi: 10.1091/mbc.E10-09-0760

Haince, J.-F., Rouleau, M., and Poirier, G. G. (2006). Transcription. Gene expression needs a break to unwind before carrying on. Science 312, 1752-1753. doi: 10.1126/science. 1129808

Han, S., Liu, Y., and Chang, A. (2007). Cytoplasmic Hsp70 promotes ubiquitination for endoplasmic reticulum-associated degradation of a misfolded mutant of the yeast plasma membrane ATPase, PMA1. J. Biol. Chem. 282, 26140-26149. doi: 10.1074/jbc.M701969200

Hartl, F. U., Bracher, A., and Hayer-Hartl, M. (2011). Molecular chaperones in protein folding and proteostasis. Nature 475, 324-332. doi: 10.1038/nature10317

Heck, J. W., Cheung, S. K., and Hampton, R. Y. (2010). Cytoplasmic protein quality control degradation mediated by parallel actions of the E3 ubiquitin ligases Ubr1 and San1. Proc. Natl. Acad. Sci. U.S.A. 107, 1106-1111. doi: 10.1073/pnas.0910591107

Houck, S. A., Singh, S., and Cyr, D. M. (2012). Cellular responses to misfolded proteins and protein aggregates. Methods Mol. Biol. 832, 455-461. doi: 10.1007/978-1-61779-474-2_32

Hundley, H. A., Walter, W., Bairstow, S., and Craig, E. A. (2005). Human Mpp11 J protein: ribosome-tethered molecular chaperones are ubiquitous. Science 308, 1032-1034. doi: 10.1126/science.1109247

Jentsch, S., and Rumpf, S. (2007). Cdc48 (p97): a "molecular gearbox" in the ubiquitin pathway? Trends Biochem. Sci. 32, 6-11. doi: 10.1016/j.tibs.2006.11.005

Jiang, J., Maes, E. G., Taylor, A. B., Wang, L., Hinck, A. P., Lafer, E. M., et al. (2007). Structural basis of J cochaperone binding and regulation of Hsp70. Mol. Cell 28, 422-433. doi: 10.1016/j.molcel.2007.08.022

Kälin, N., Claass, A., Sommer, M., Puchelle, E., and Tümmler, B. (1999). DeltaF508 CFTR protein expression in tissues from patients with cystic fibrosis. J. Clin. Invest. 103, 1379-1389. doi: 10.1172/JCI5731

Kampinga, H. H., and Craig, E. A. (2010). The HSP70 chaperone machinery: J proteins as drivers of functional specificity. Nat. Rev. Mol. Cell Biol. 11, 579-592. doi: $10.1038 / \mathrm{nrm} 2941$

Kaufman, R. J., Scheuner, D., Schröder, M., Shen, X., Lee, K., Liu, C. Y., et al. (2002). The unfolded protein response in nutrient sensing and differentiation. Nat. Rev. Mol. Cell Biol. 3, 411-421. doi: 10.1038/nrm829 
Kim, Y. E., Hipp, M. S., Bracher, A., Hayer-Hartl, M., and Hartl, F. U. (2013). Molecular chaperone functions in protein folding and proteostasis. Annu. Rev. Biochem. 82, 323-355. doi: 10.1146/annurev-biochem-060208-092442

Konieczny, I., and Zylicz, M. (1999). Role of bacterial chaperones in DNA replication. Genet. Eng. (N.Y). 21, 95-111. doi: 10.1007/978-1-4615-4707-5_6

Lee, A. S. (1992). Mammalian stress response: induction of the glucoseregulated protein family. Curr. Opin. Cell Biol. 4, 267-273. doi: 10.1016/09550674(92)90042-B

Li, J., Qian, X., and Sha, B. (2003). The crystal structure of the yeast Hsp40 Ydj1 complexed with its peptide substrate. Structure 11, 1475-1483. doi: 10.1016/j.str.2003.10.012

Lüders, J., Demand, J., and Höhfeld, J. (2000). The ubiquitin-related BAG-1 provides a link between the molecular chaperones Hsc70/Hsp70 and the proteasome. J. Biol. Chem. 275, 4613-4617. doi: 10.1074/jbc.275.7.4613

Lukacs, G. L., and Verkman, A. S. (2012). CFTR: folding, misfolding and correcting the? $\Delta$ F508 conformational defect. Trends Mol. Med. 18, 81-91. doi: 10.1016/j.molmed.2011.10.003

Malinovska, L., Kroschwald, S., Munder, M. C., Richter, D., and Alberti, S. (2012). Molecular chaperones and stress-inducible protein-sorting factors coordinate the spatiotemporal distribution of protein aggregates. Mol. Biol. Cell 23, 3041-3056. doi: 10.1091/mbc.E12-03-0194

Meacham, G. C., Patterson, C., Zhang, W., Younger, J. M., and Cyr, D. M. (2001). The Hsc70 co-chaperone CHIP targets immature CFTR for proteasomal degradation. Nat. Cell Biol. 3, 100-105. doi: 10.1038/35050509

Mehnert, M., Sommermeyer, F., Berger, M., Kumar Lakshmipathy, S., Gauss, R., Aebi, M., et al. (2015). The interplay of $\mathrm{Hrd} 3$ and the molecular chaperone system ensures efficient degradation of malfolded secretory proteins. Mol. Biol. Cell 26, 185-194. doi: 10.1091/mbc.E14-07-1202

Miller, S. B. M., Ho, C.-T., Winkler, J., Khokhrina, M., Neuner, A., Mohamed, M. Y. H., et al. (2015). Compartment-specific aggregases direct distinct nuclear and cytoplasmic aggregate deposition. EMBO J. 34, 778-797. doi: $10.15252 / \mathrm{embj} .201489524$

Morimoto, R. I. (2008). Proteotoxic stress and inducible chaperone networks in neurodegenerative disease and aging. Genes Dev. 22, 1427-1438. doi: $10.1101 /$ gad. 1657108

Murray, A. W. (2004). Recycling the cell cycle: cyclins revisited. Cell 116, 221-234. doi: 10.1016/S0092-8674(03)01080-8

Nagai, S., Davoodi, N., and Gasser, S. M. (2011). Nuclear organization in genome stability: SUMO connections. Cell Res. 21, 474-485. doi: 10.1038/cr.2011.31

Nakatsukasa, K., Huyer, G., Michaelis, S., and Brodsky, J. L. (2008). Dissecting the ER-associated degradation of a misfolded polytopic membrane protein. Cell 132, 101-112. doi: 10.1016/j.cell.2007.11.023

Nollen, E. A., Salomons, F. A., Brunsting, J. F., van der Want, J. J., Sibon, O. C., and Kampinga, H. H. (2001). Dynamic changes in the localization of thermally unfolded nuclear proteins associated with chaperone-dependent protection. Proc. Natl. Acad. Sci. U.S.A. 98, 12038-12043. doi: 10.1073/pnas.201112398

Okiyoneda, T., Barrière, H., Bagdány, M., Rabeh, W. M., Du, K., Höhfeld, J., et al. (2010). Peripheral protein quality control removes unfolded CFTR from the plasma membrane. Science 329, 805-810. doi: 10.1126/science.1191542

Park, S.-H., Kukushkin, Y., Gupta, R., Chen, T., Konagai, A., Hipp, M. S., et al. (2013). PolyQ proteins interfere with nuclear degradation of cytosolic proteins by sequestering the Sislp chaperone. Cell 154, 134-145. doi: 10.1016/j.cell.2013.06.003

Prasad, R., Kawaguchi, S., and Ng, D. T. W. (2010). A nucleus-based quality control mechanism for cytosolic proteins. Mol. Biol. Cell 21, 2117-2127. doi: 10.1091/mbc.E10-02-0111

Ravid, T., Kreft, S. G., and Hochstrasser, M. (2006). Membrane and soluble substrates of the Doa10 ubiquitin ligase are degraded by distinct pathways. EMBO J. 25, 533-543. doi: 10.1038/sj.emboj.7600946
Richly, H., Rocha-Viegas, L., Ribeiro, J. D., Demajo, S., Gundem, G., Lopez-Bigas, N., et al. (2010). Transcriptional activation of polycombrepressed genes by ZRF1. Nature 468, 1124-1128. doi: 10.1038/nature 09574

Russell, S. J., Steger, K. A., and Johnston, S. A. (1999). Subcellular localization, stoichiometry, and protein levels of $26 \mathrm{~s}$ proteasome subunits in yeast. J. Biol. Chem. 274, 21943-21952. doi: 10.1074/jbc.274.31. 21943

Schelhaas, M., Malmström, J., Pelkmans, L., Haugstetter, J., Ellgaard, L., Grünewald, K., et al. (2007). Simian Virus 40 depends on ER protein folding and quality control factors for entry into host cells. Cell 131, 516-529. doi: 10.1016/j.cell.2007.09.038

Scheuner, D., Song, B., McEwen, E., Liu, C., Laybutt, R., Gillespie, P., et al. (2001). Translational control is required for the unfolded protein response and in vivo glucose homeostasis. Mol. Cell 7, 1165-1176. doi: 10.1016/S10972765(01)00265-9

Shiber, A., Breuer, W., Brandeis, M., and Ravid, T. (2013). Ubiquitin conjugation triggers misfolded protein sequestration into quality control foci when Hsp70 chaperone levels are limiting. Mol. Biol. Cell 24, 2076-2087. doi: 10.1091/mbc.E13-01-0010

Swain, J. F., Dinler, G., Sivendran, R., Montgomery, D. L., Stotz, M., and Gierasch, L. M. (2007). Hsp70 chaperone ligands control domain association via an allosteric mechanism mediated by the interdomain linker. Mol. Cell 26, 27-39. doi: 10.1016/j.molcel.2007.02.020

Tsai, B., Ye, Y., and Rapoport, T. A. (2002). Retro-translocation of proteins from the endoplasmic reticulum into the cytosol. Nat. Rev. Mol. Cell Biol. 3, 246-255. doi: $10.1038 / \mathrm{nrm} 780$

Turner, G. C., and Varshavsky, A. (2000). Detecting and measuring cotranslational protein degradation in vivo. Science 289, 2117-2120. doi: 10.1126/science.289.5487.2117

Verma, R., Oania, R. S., Kolawa, N. J., and Deshaies, R. J. (2013). Cdc48/p97 promotes degradation of aberrant nascent polypeptides bound to the ribosome Elife 2:e00308. doi: 10.7554/eLife.00308

Wang, F., Durfee, L. A., and Huibregtse, J. M. (2013). A cotranslational ubiquitination pathway for quality control of misfolded proteins. Mol. Cell 50, 368-378. doi: 10.1016/j.molcel.2013.03.009

Westhoff, B., Chapple, J. P., van der Spuy, J., Höhfeld, J., and Cheetham, M. E. (2005). HSJ1 is a neuronal shuttling factor for the sorting of chaperone clients to the proteasome. Curr. Biol. 15, 1058-1064. doi: 10.1016/j.cub.2005. 04.058

Willmund, F., del Alamo, M., Pechmann, S., Chen, T., Albanèse, V., Dammer, E. B., et al. (2013). The cotranslational function of ribosomeassociated Hsp70 in eukaryotic protein homeostasis. Cell 152, 196-209. doi: 10.1016/j.cell.2012.12.001

Younger, J. M., Chen, L., Ren, H.-Y., Rosser, M. F. N., Turnbull, E. L., Fan, C.-Y., et al. (2006). Sequential quality-control checkpoints triage misfolded cystic fibrosis transmembrane conductance regulator. Cell 126, 571-582. doi: 10.1016/j.cell.2006.06.041

Conflict of Interest Statement: The authors declare that the research was conducted in the absence of any commercial or financial relationships that could be construed as a potential conflict of interest.

Copyright (c) 2015 Dekker, Kampinga and Bergink. This is an open-access article distributed under the terms of the Creative Commons Attribution License (CC BY). The use, distribution or reproduction in other forums is permitted, provided the original author(s) or licensor are credited and that the original publication in this journal is cited, in accordance with accepted academic practice. No use, distribution or reproduction is permitted which does not comply with these terms. 